\title{
Differential regulation of cell death programs in males and females by Poly (ADP-Ribose) Polymerase-1 and $17 \beta$ estradiol
}

\author{
NR Jog ${ }^{1}$ and R Caricchio, ${ }^{*}$
}

Cell death can be divided into the anti-inflammatory process of apoptosis and the pro-inflammatory process of necrosis. Necrosis, as apoptosis, is a regulated form of cell death, and Poly-(ADP-Ribose) Polymerase-1 (PARP-1) and ReceptorInteracting Protein (RIP) 1/3 are major mediators. We previously showed that absence or inhibition of PARP-1 protects mice from nephritis, however only the male mice. We therefore hypothesized that there is an inherent difference in the cell death program between the sexes. We show here that in an immune-mediated nephritis model, female mice show increased apoptosis compared to male mice. Treatment of the male mice with estrogens induced apoptosis to levels similar to that in female mice and inhibited necrosis. Although PARP-1 was activated in both male and female mice, PARP-1 inhibition reduced necrosis only in the male mice. We also show that deletion of RIP-3 did not have a sex bias. We demonstrate here that male and female mice are prone to different types of cell death. Our data also suggest that estrogens and PARP-1 are two of the mediators of the sex-bias in cell death. We therefore propose that targeting cell death based on sex will lead to tailored and better treatments for each gender. Cell Death and Disease (2013) 4, e758; doi:10.1038/cddis.2013.251; published online 8 August 2013

Subject Category: Experimental Medicine

Several studies have shown that the response of males and females to stress differs. ${ }^{1-3}$ It is generally believed that females respond to infection and trauma with increased antibody production, which may also increase their risk of developing autoimmune diseases. ${ }^{1-4}$ Higher prevalence of autoimmune diseases such as systemic lupus erythematosus (SLE), Sjogren's syndrome and rheumatoid arthritis (RA) in females supports this notion. ${ }^{1,5,6}$ Interestingly, inflammation is usually considered more severe in males resulting in an increased mortality. $3,4,7$

Several inflammatory diseases show differences in severity and outcome between the two sexes. Males have larger stroke areas and more severe sepsis. Acute respiratory distress syndrome (ARDS) shows rapid destruction of lung tissue in males, and estrogens delay the damage..$^{8-10}$ However, some autoimmune diseases such as ANCA-associated vasculitis and $\lg A$ nephropathy also show higher incidence in males. ${ }^{1,11}$ Moreover, although SLE is more prevalent in females, renal disease in SLE is often more severe in males and has a poor prognosis. ${ }^{12-14}$

Du et al..$^{15}$ reported innate gender differences in response to cytotoxic agents and programmed cell death. XY neurons were more susceptible to nitrosative stress and exhibited a proclivity towards an apoptosis-inducing factor-dependent pathway, while XX neurons were more susceptible to apoptosis-inducing agents and show a proclivity towards the cytochrome $c$-dependent pathway. The authors showed that these gender-dependent differences were due to the incapacity of $X Y$ neurons to maintain intracellular levels of reduced glutathione: a prominent defense against oxidative stress. Li et al. ${ }^{16}$ also demonstrated an intrinsic female neuroprotection in hippocampus organotypic slice cultures after oxygen and glucose deprivation, suggesting again a sexually dimorphic use of cell death pathways.

During physiological stress, the activated immune system secretes pro-inflammatory cytokines in addition to the activation of NADPH oxidase and generation of reactive oxygen species. This initial response leads to increased cell death, which further directs the immune response. Several forms of cell death have been identified. Apoptosis, traditionally considered a form of programmed cell death, is anti-inflammatory whereas necrosis and necroptosis are pro-inflammatory. ${ }^{17-19}$ Necrosis is also accepted as a regulated form of cell death, and specific signaling pathways have been identified. One such pathway is regulated by Poly-(ADP-Ribose) Polymerase-1 (PARP-1). ${ }^{17,18}$ PARP-1 is a DNA repair enzyme whose enzymatic activity is induced by DNA damage. ${ }^{20}$ PARP-1 and the induction of necrosis have been involved in several pathogenic processes, from stroke to rheumatoid arthritis. ${ }^{21}$ We showed previously that increased oxidative stress and the resultant DNA damage lead to hyperactivation of PARP-1 in the kidneys during immunemediated nephritis. ${ }^{22}$ Inhibition or absence of PARP-1 results in reduced renal inflammation and better survival of mice.

\footnotetext{
${ }^{1}$ Rheumatology Section, Department of Medicine, Temple Autoimmunity Center, Temple University School of Medicine, Philadelphia, PA, USA

${ }^{*}$ Corresponding author: R Caricchio, Rheumatology Section, Department of Medicine, Temple Autoimmunity Center, Temple University School of Medicine, 1154 MERB, 3500 North. Broad Street, Philadephia, PA 19140, USA. Tel: 215707 4639; Fax: 215707 3508; Email: roc@ temple.edu

Keywords: apoptosis; $17 \beta$ estradiol; female; male; necrosis; PARP-1

Abbreviations: E2, $17 \beta$ estradiol; ER $\alpha$, estrogen receptor $\alpha$; KS, Klinefelter's syndrome; NZW, New Zealand White; PARP-1, Poly (ADP-Ribose) Polymerase-1; PAR, Poly (ADP-Ribose) Polymers; RIP-1, Receptor-Interacting Protein Kinase 1; Z-DEVD-FMK, Benzyloxycarbonyl-Asp(OMe)-Glu(OMe)-Val-Asp(OMe)-fluoromethylketone Received 01.10.12; revised 02.5.13; accepted 06.6.13; Edited by Y Shi
} 
Interestingly, protection conferred by absence or inhibition of PARP-1 showed a sex-bias, with only male mice being protected.

These results suggest that the differences in stress response in males and females may be due to a difference in the cell death program in the two sexes. We therefore hypothesized that males are prone to PARP-1 necrosis, whereas females are prone to caspase-dependent apoptosis.

It has been reported that estrogens can enhance the resistance to oxidative stress. ${ }^{23}$ Furthermore, the sex-bias we observed in the activity of PARP-1 may be partially explained by the ability of PARP-1 to interact with estradiol and estrogen receptor $\alpha(E R \alpha)$. ER $\alpha$ complex stabilizes PARP-1 binding to DNA and diminishes the capacity of PARP-1 to hyperactivate. ${ }^{24}$ We showed previously that treatment of male mice with $17 \beta$ Estradiol (E2) conferred protection against immune-mediated nephritis. Here we aimed at determining whether estrogens had a role in defining the susceptibility of male and female cells to different forms of cell death.

\section{Results}

PARP-1 is activated in both male and female mice during nephritis. We previously observed a sex bias in the activity of PARP-1. To determine whether this bias was due to the activation of PARP-1 in only one sex, we determined PARP-1 activation in male and female mice during lupus nephritis. The comparison of kidney disease between male and female mice is often challenging due to the sex-bias of disease severity in various mouse models of lupus. We took advantage of the new model developed by Dr. Davidson's group. ${ }^{25}$ We used kidneys from NZW/BXSB F1 male and female mice. The NZW/BXSB F1 male mice spontaneously develop lupus, whereas the female mice develop similar disease severity when injected with Interferon alpha (IFN $\alpha$ ).
The kidney sections from male and female mice were stained for Poly (ADP-Ribose) Polymers (PARs) as a measure of PARP-1 activation. In Figures $1 a$ and $b$, we show that PARP1 was activated in male and also in female mice that were injected with IFN $\alpha$.

PARs are a product of poly (ADP-ribosyl)-ation, and several members of the PARP family can mediate this reaction. ${ }^{20}$ To further demonstrate the role of PARP-1 we used nephrotoxic serum (NTS)-induced nephritis (NTN) as a model of immunemediated nephritis. ${ }^{22}$ In this model mice are injected with serum (NTS) from a sheep immunized with mouse glomeruli. The NTN is characterized by initial heterologous phase where the injected antibodies (NTS) bind glomerular antigens, fix complement and initiate an inflammatory response. An advantage of the NTN model is that it allows to 'bypass' the upstream events during systemic autoimmunity that lead to loss of tolerance and generation of auto-antibodies. ${ }^{26,27}$ Moreover, recent evidence suggests that, irrespective of the upstream events leading to immune-mediated nephritis, the downstream mechanisms involved in renal tissue damage are similar. ${ }^{26,27}$ NTN in PARP-1-deficient mice demonstrates that PARP-1 is responsible for the majority of poly (ADP-ribosyl)ation in the nephritic kidney, and therefore PAR accumulation can be used as a measure of PARP-1 activation (Figure 1c). Moreover the data obtained from this model can be applied to other immune-mediated nephropathies including lupus nephritis.

Absence of PARP-1 activity rescues from necrotic cell death. Sex hormones are known to have an important role in the immune system. However, evidence suggests that the genetic differences between males and females may also contribute to the observed differences in phenotype between the two sexes. ${ }^{28,29}$ To determine the differences in response to necrotic stimuli due to sex-chromosome component, we induced cell death in bone marrow-derived macrophages a

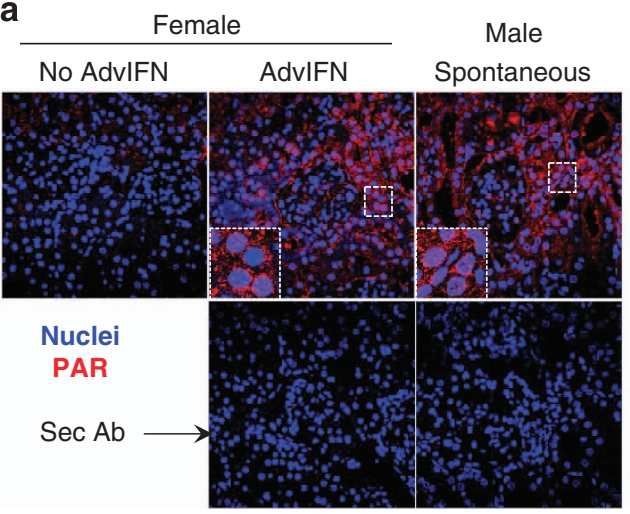

b

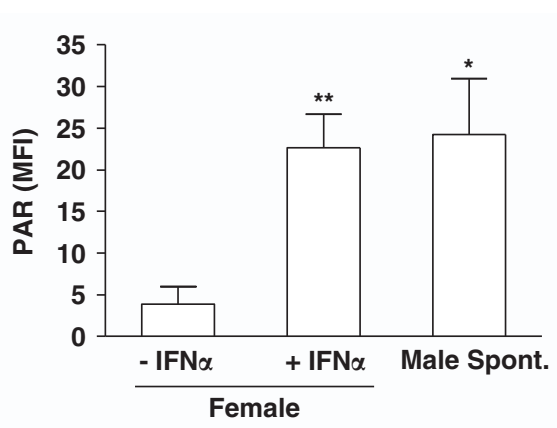

C

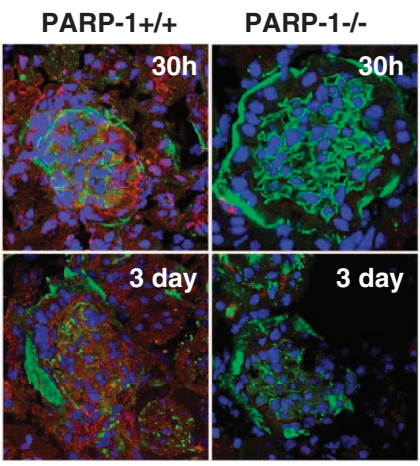

Figure 1 PARP-1 is activated in both male and female mice during nephritis. (a) Kidney sections from (NZWXBXSB)F1 mice were stained with DAPI to identify nuclei and with anti-PARs to identify PARP-1 enzymatic activity; bottom panels shows secondary antibody only. Female mice were injected with AdvIFN $\alpha$ at 8 weeks and were 22 weeks old at the time of the staining. The male mice were 18 weeks old. The figures are representative of six mice. (b) Fluorescence intensities of red channel were measured using ImageJ software. Fluorescence intensities of the secondary controls were subtracted from the stained sections. An average of 5-7 fields was calculated for each mouse. Data are mean \pm S.E.M. ${ }^{*} P<0.05,{ }^{*} P<0.005$. (c) NTN was induced in PARP-1 + / + or PARP-1 $-/$ - mice by injecting NTS. Kidneys were collected $30 \mathrm{~h}$ and 3 days after induction of NTN and stained with anti-PAR, Fluoroscein-conjugated anti-Complement C 3 and DAPI. PARs were visualized by Rhodamine-conjugated secondary antibody. The figure shows that although C3 deposition in PARP-1 - / - kidneys was same as PARP-1 $/$ / kidneys, PAR accumulation was absent in PARP-1 - / - kidneys. These data show that PARP-1 is responsible for the majority of poly (ADP-ribosyl)-ation in the kidney during nephritis and therefore PARs can be used as a measure of PARP-1 activity in the kidney 
(BMDM) from male and female mice. We used BMDM because our previously published data with bone marrow chimera show that PARP-1 absence is required in both immune and non-immune compartments for protection against immune-mediated nephritis. ${ }^{22}$ The results suggested that not only intrinsic renal cells but also bone marrowderived hematopoietic cells undergo PARP-1-dependent cell death during nephritis. Figure $2 \mathrm{a}$ shows that lower concentrations of hydrogen peroxide induced apoptosis, whereas higher concentrations induced necrosis as determined by Annexin $V$ and $7 A A D$ positivity. Figure $2 a$ also shows that the dose required for inducing cell death in male and female cells was the same, suggesting that male and female cells do not differ in their susceptibility to $\mathrm{H}_{2} \mathrm{O}_{2}$-induced apoptosis or

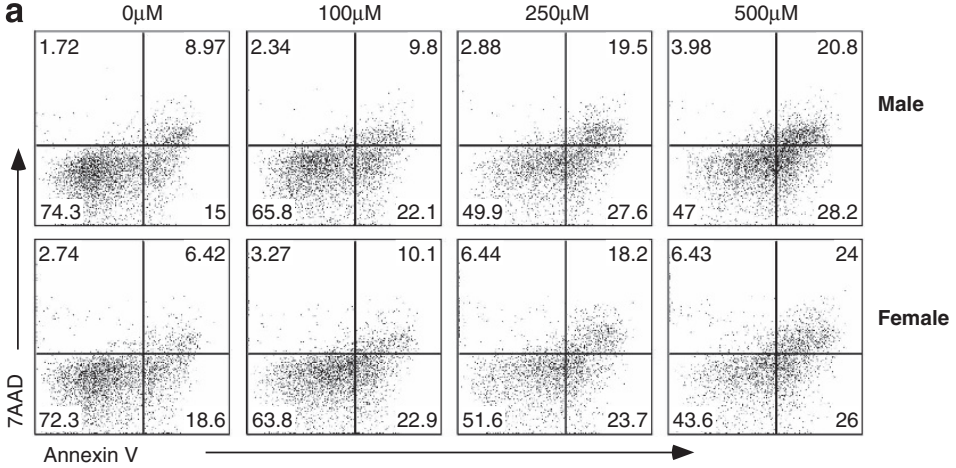

b

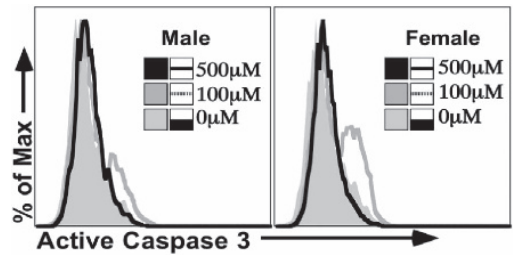

C

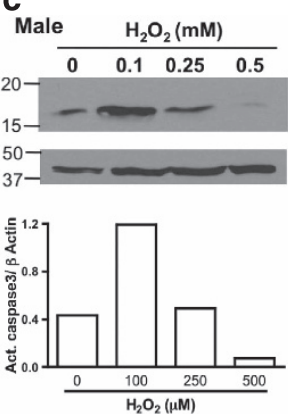

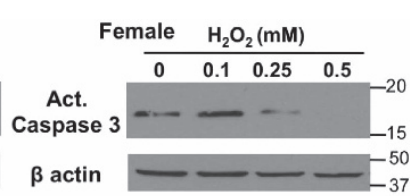

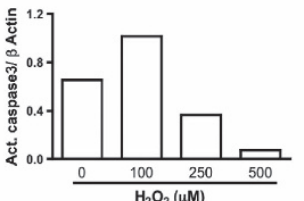

d
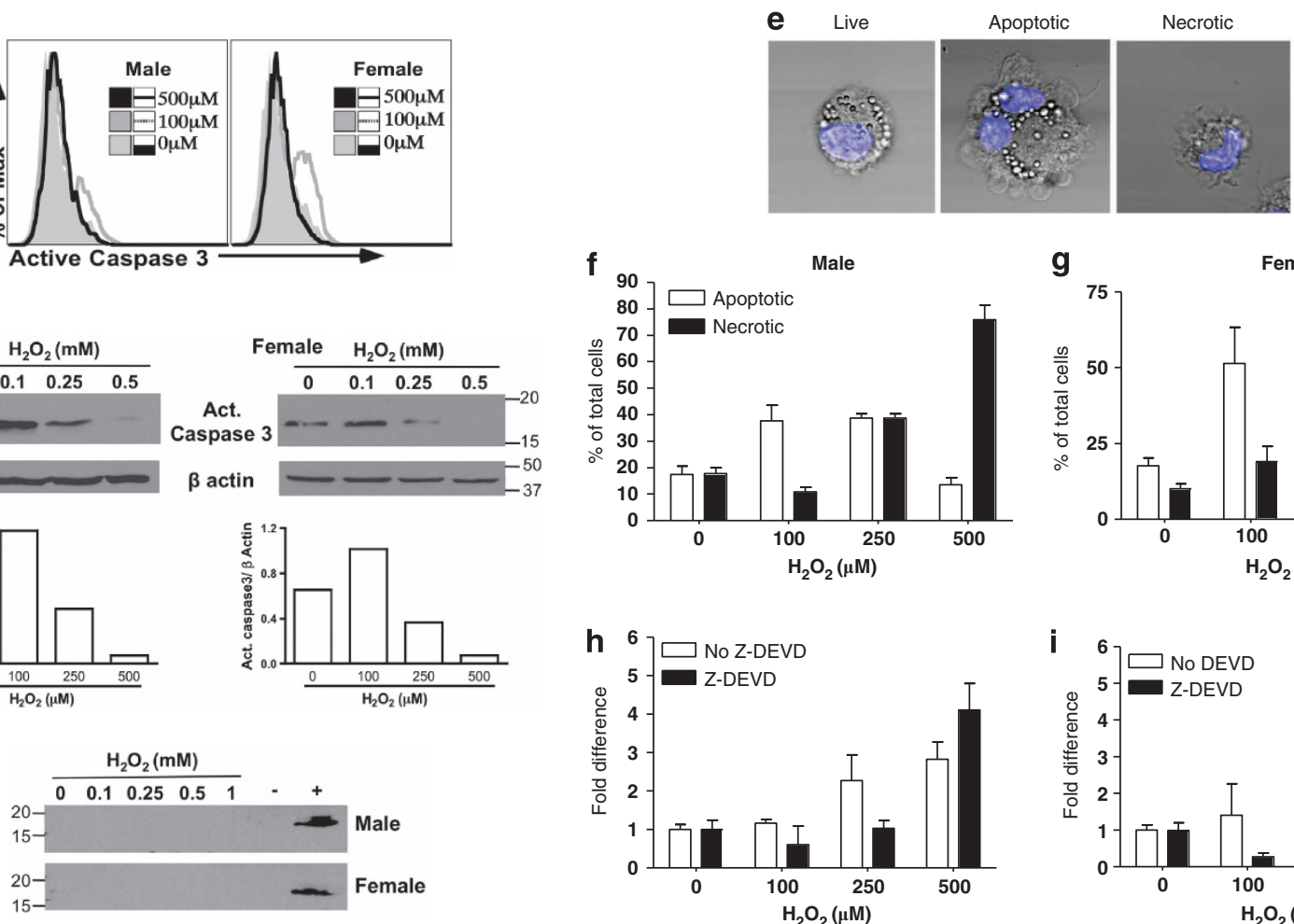

f
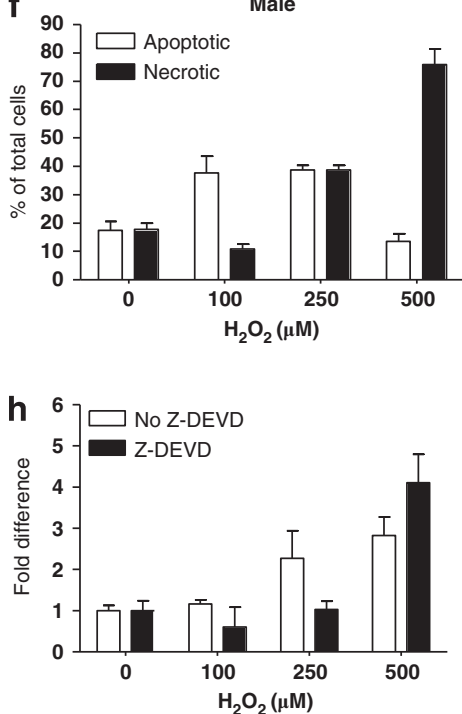

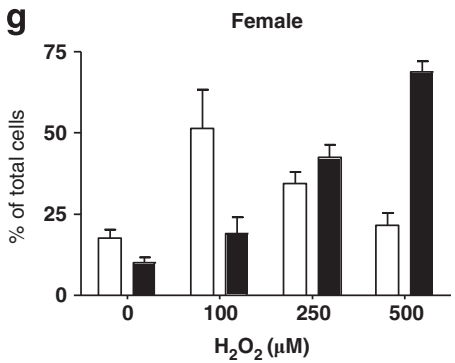

i

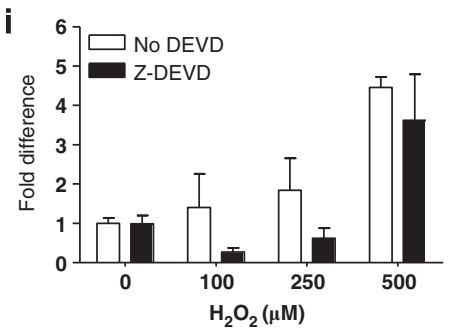

Figure 2 Male and female cells show similar susceptibility to $\mathrm{H}_{2} \mathrm{O}_{2}$-induced cell death. BMDM from male and female 129s mice were stimulated with various concentrations of $\mathrm{H}_{2} \mathrm{O}_{2}$ for $2 \mathrm{~h}$. (a) The cells were harvested and stained for FITC conjugated Annexin V and 7AAD, and acquired on FACS Canto. One representative of two independent cultures is shown. (b) BMDMs were stimulated, harvested, fixed, permeabilized and stained with PE-conjugated active caspase-3 antibodies. Cells were acquired on a FACS Canto and analyzed using FlowJo. One representative of four independent cultures is shown. (c) BMDMs were stimulated with increasing concentrations of $\mathrm{H}_{2} \mathrm{O}_{2}$ as in (a). Cells were lysed after $2 \mathrm{~h}$ stimulation. Western blot analysis shows that higher concentration of $\mathrm{H}_{2} \mathrm{O}_{2}$-induced necrosis as seen by absence of active caspase-3 in cell lysates (upper panel). Lower panel shows the ratios of band density of active caspase-3 to $\beta$ actin band density, which was used as a loading control. (d) Active caspase-3 was not detected in concentrated culture supernatants from BMDMs treated with $\mathrm{H}_{2} \mathrm{O}_{2}$. Upper panel: concentrated supernatants from male BMDMs, lower panel: concentrated supernatants from female BMDMs. ' - ' and '+ ' represent lysates of untreated Jurkat cells ( - ) or Jurkat cells treated with camptothecin (+). BMDMs from male and female were labeled with Hoechst 33342 and stimulated with $\mathrm{H}_{2} \mathrm{O}_{2}$ for $2 \mathrm{~h}$. Cells were fixed and imaged. Apoptotic and necrotic cells were counted. (e) Representative examples of live, apoptotic and necrotic cells are shown. Cells were identified as apoptotic or necrotic according to Figure 2e. Stimulation with $500 \mu_{m} \mathrm{H}_{2} \mathrm{O}_{2}$-induced necrosis in both male (f) and female BMDMs (g). Caspase-3 inhibition by Z-DEVD-FMK did not inhibit necrosis induced by $500 \mu \mathrm{m} \mathrm{H}_{2} \mathrm{O}_{2}$ in either male (h) or female (i) BMDMs. The data show that both male and female cells show similar susceptibility to $\mathrm{H}_{2} \mathrm{O}_{2}$-induced cell death and higher concentrations of $\mathrm{H}_{2} \mathrm{O}_{2}$ induce necrosis in both male and female cells 
necrosis. Intracellular active caspase-3 further confirmed that lower concentrations of $\mathrm{H}_{2} \mathrm{O}_{2}$ induced apoptosis, whereas high doses induced necrosis (Figure $2 \mathrm{~b}$ ). We also performed western blots on whole-cell lysates from male and female BMDMs stimulated with increasing concentrations of $\mathrm{H}_{2} \mathrm{O}_{2}$. Similar to our data with flow cytometry, western blot analysis shows that caspase- 3 is activated when BMDMs are stimulated with lower concentrations, but not with higher concentrations $(500 \mu \mathrm{m})$ of $\mathrm{H}_{2} \mathrm{O}_{2}$ (Figure 2c). Late apoptotic cells may release active caspase- 3 in the culture supernatant as a result of the loss of membrane integrity. To demonstrate that the percentage of late apoptotic cells in our system was negligible, we determined the levels of active caspase- 3 in the supernatants. We did not detect any active caspase-3 released in the supernatants by dying cells (Figure $2 \mathrm{~d}$ ). We have confirmed by confocal microscopy that increasing doses of $\mathrm{H}_{2} \mathrm{O}_{2}$ induce apoptosis and necrosis respectively (Figures $2 \mathrm{e}-\mathrm{g}$ ) and that under these circumstances the Caspase-3 inhibitor only interferes with apoptosis (Figures $2 \mathrm{~h}$ and i). These data show that both male and female cells show similar susceptibility to $\mathrm{H}_{2} \mathrm{O}_{2}$-induced cell death and higher concentrations of $\mathrm{H}_{2} \mathrm{O}_{2}$ induces primary necrosis in both male and female cells. Finally, Figure 2 also suggests that, as described previously, necrotic cells can also be Annexin V-positive, ${ }^{30,31}$ however, caspase-3 activation is specific for apoptosis.

To further determine whether necrosis induced by $\mathrm{H}_{2} \mathrm{O}_{2}$ is PARP-1-dependent, male and female BMDMs from wild-type or PARP-1-deficient mice were stimulated with $\mathrm{H}_{2} \mathrm{O}_{2}$. Figures $3 a-d$ shows that while PARP-1 deficiency inhibited necrosis in male cells, $\mathrm{H}_{2} \mathrm{O}_{2}$-induced cell death in female macrophages was independent of PARP-1 activation. These data are in agreement with our previous results. PARP-1 deficiency in male cells not only inhibited necrosis but also induced apoptosis, and did not change the viability. Pharmacological inhibition of PARP-1 also inhibited necrosis and increased apoptosis in male cells (not shown). The data suggest that male BMDM undergo a PARP-1-dependent cell death program. We also observed increased apoptosis in the absence of PARP-1 in female cells. Although the reason is not clear yet and requires further studies, a possibility is that in the absence of PARP-1 the DNA repair machinery is ineffective and therefore the cells are facilitated to undergo apoptotic cell death. However, as female cells do undergo necrosis, we sought to determine if the necrosis in female cells was through a newly identified cell death program: necroptosis. ${ }^{19}$ Necroptosis is dependent on ReceptorInteracting Protein Kinase 1 and 3 (RIP-1 and RIP-3). Necroptosis can be inhibited by either a small-molecule inhibitor of RIP-1, Necrostatin-1, ${ }^{32}$ or by genetic deletion of RIP-3. To determine whether female cells preferentially undergo necroptosis, we stimulated BMDMs from RIP-3-sufficient or RIP-3-deficient mice with $\mathrm{H}_{2} \mathrm{O}_{2}$. Figures $3 e-h$ show that necrotic death induced by $\mathrm{H}_{2} \mathrm{O}_{2}$ in both male and female cells is independent of RIP-3 activation. Inhibition of RIP-1 by Necrostatin-1 also did not alter cell death in male or female cells (not shown). These data are in line with observations from Vanden Berghe et al. ${ }^{33}$ that $\mathrm{H}_{2} \mathrm{O}_{2}$-induced cell death is independent of RIP-1/3 activation.

Estradiol inhibits necrotic cell death. Mabley et al. ${ }^{24}$ showed that PARP-1 interacts with ER $\alpha$ and estrogen, and due to this interaction, estrogen may inhibit PARP-1 activity. Moreover, we also showed that estradiol treatment of male mice conferred protection against immune-mediated nephritis, similar to PARP-1 inhibition. Therefore, to determine whether estrogens can also inhibit necrotic cell death by inhibiting PARP-1 activity, we induced necrosis in male and female cells in the presence or absence of $17 \beta$ estradiol (E2). Figures $4 \mathrm{a}$ and $\mathrm{d}$ show that E2 inhibited necrotic cell death in both male and female cells. Although E2 treatment rescued female macrophages from both necrosis and apoptosis, in males E2 only inhibited necrosis (Figures $4 b$ and e). We further determined the ability of estrogens to inhibit PARP-1
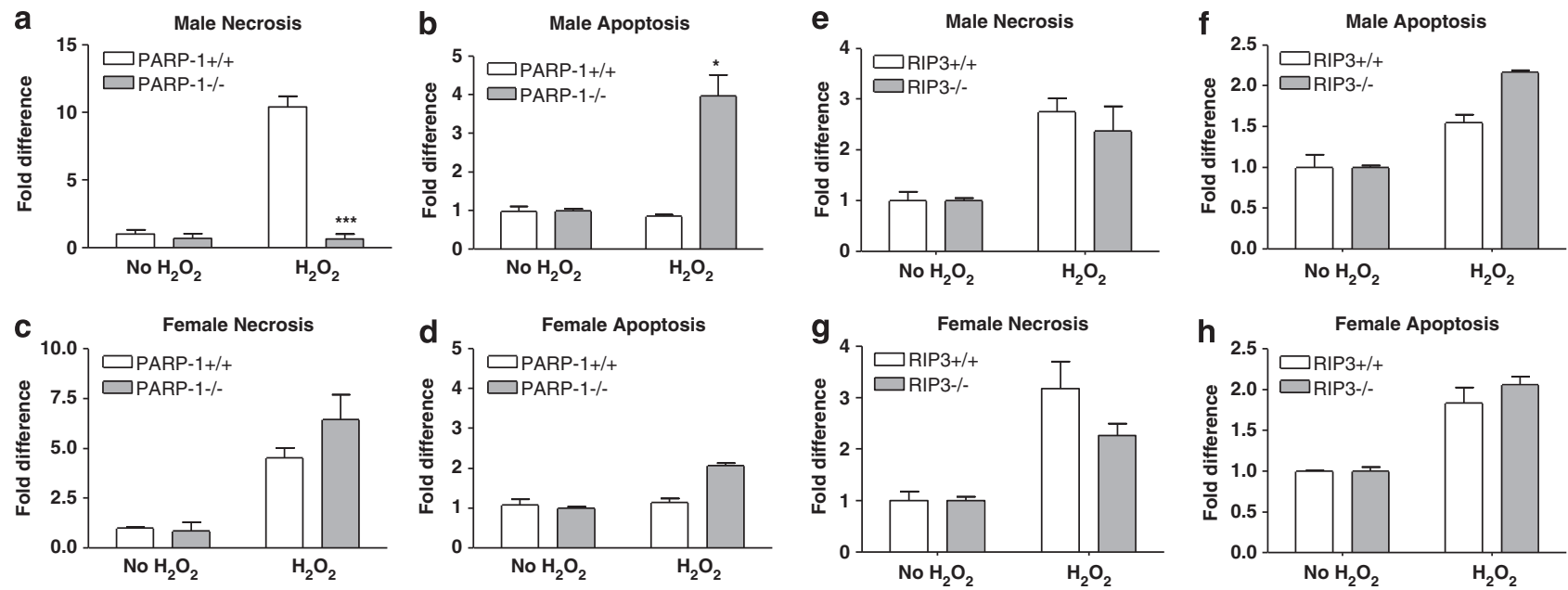

Figure $3 \quad \mathrm{H}_{2} \mathrm{O}_{2}$-induced necrosis is PARP-1 dependent in male BMDM. BMDM from male and female 129 s mice or 129 PARP-1 - / - mice (a-d); B6 or RIP-3 - / mice (e-h) were stimulated with $\mathrm{H}_{2} \mathrm{O}_{2}$. The cells were collected and stained for FITC conjugated Annexin V and Propidium lodide/7AAD, and acquired on FACS Canto. Data are mean \pm S.E.M., representative of two independent cultures is shown. The data show that $\mathrm{H}_{2} \mathrm{O}_{2}$-induced cell death in male cells is dependent on PARP-1 activation while necrosis in both males and females is independent of RIP-3 activation. ${ }^{\star} P<0.05,{ }^{* \star *} P<0.0005$ 

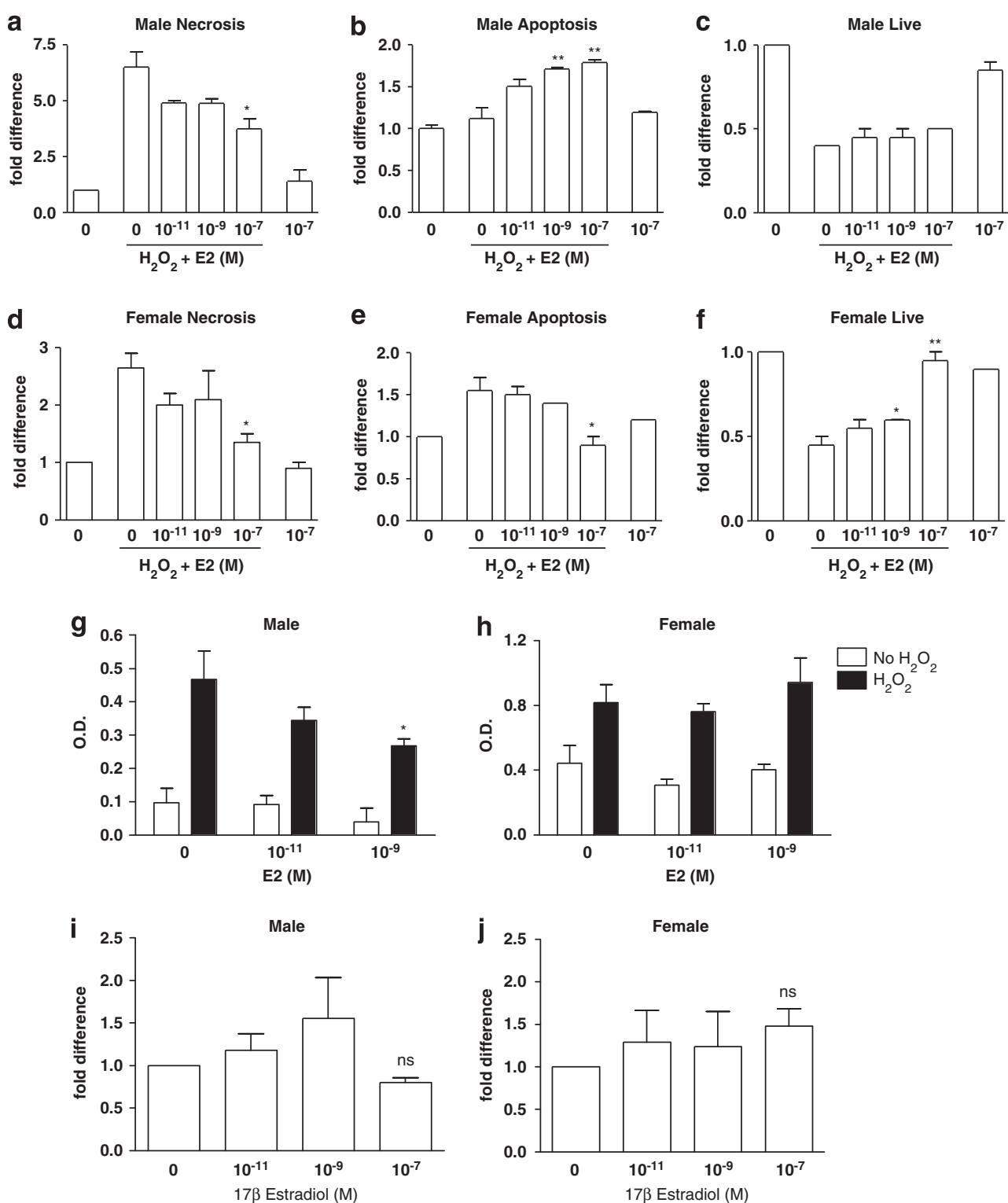

Figure $4 \quad 17 \beta$ Estradiol influences PARP-1 activation and cell death in male and female cells. (a-f) BMDM from male and female 129s mice were pre-treated with various concentrations of $\mathrm{E} 2$ for $3 \mathrm{~h}$ followed by 2 hours stimulation with $\mathrm{H}_{2} \mathrm{O}_{2}$. The cells were harvested and stained for FITC-conjugated Annexin V and Propidium lodide/7AAD. Results were acquired on FACS Canto. The data show that E2 rescues female cells from death, whereas it shifts cell death program to apoptosis in male cells. Data are mean \pm S.E.M.; representative of four independent experiments is shown. ( $\mathbf{g}$ and $\mathbf{h}$ ) BMDMs were seeded in 96-well plates and pre-treated with E2 for $3 \mathrm{~h}$. Following stimulation with $\mathrm{H}_{2} \mathrm{O}_{2}$, PARP-1 activity in the cells was determined by a colorimetric assay as described in Materials and Methods. The data show that E2 inhibits PARP-1 activity in male $(\mathbf{g})$ but not in female cells $(\mathbf{h})$. Data are mean \pm S.E.M. of triplicates. One representative of three experiments is shown. (i and $\mathbf{j})$ ER $\alpha$ mRNA levels were determined in BMDMs treated with E2 and stimulated with $\mathrm{H}_{2} \mathrm{O}_{2}$ as in (a-f) by quantitative real-time PCR. Relative mRNA level calculated from a standard graph was normalized to mRNA levels in untreated cells. The data show that E2 treatment does not alter ER $\alpha$ expression in male (i) or female (j) cells. Data are mean \pm S.E.M., representative of six mice. ${ }^{*} P<0.05,{ }^{* *} P<0.005$

activity in male and female BMDMs stimulated with $\mathrm{H}_{2} \mathrm{O}_{2}$. Figure $4 \mathrm{~g}$ shows that E2 inhibited PARP-1 activity in male cells in a dose-dependent manner. As expected, E2 treatment did not inhibit PARP-1 activity in female BMDMs upon $\mathrm{H}_{2} \mathrm{O}_{2}$ treatment (Figure 4h). Instead PARP-1 inhibitor reduced PARP-1 activity in both male and female BMDMs (not shown).

Estrogens mediate their effect through two receptors: Estrogen Receptor $\alpha(E R \alpha)$ and Estrogen Receptor $\beta(\operatorname{ER} \beta)$. The level of expression of estrogen receptors (ERs), ER $\alpha$ and
$\mathrm{ER} \beta$, is important because a preponderance of one ER subtype over the other might change estrogen effects. ${ }^{34}$ Hall and McDonnell ${ }^{35}$ showed that ER $\beta$ can modulate ER $\alpha$ transcriptional activity, and thus the relative expression levels of the two isoforms may define the cellular responses to agonists. E2 treatment is known to modulate ER expression in a cell-type-dependent manner. In our system, the differential response of male and female cells to E2 may be due to differences in the expression levels of estrogen receptors in response to E2. We therefore determined the levels of ER $\alpha$ 
and $\mathrm{ER} \beta$ mRNA. Figures $4 \mathrm{i}$ and $\mathrm{j}$ show that $\mathrm{ER} \alpha$ levels did not statistically change in both male and female cells following E2 treatment. We also observed similar levels of ER $\beta$ expression (not shown).

Estradiol treatment of male mice induces apoptosis in kidney during nephrotoxic nephritis (NTN). To confirm the relevance of our results, we determined the effect of E2 treatment on apoptosis and necrosis in the kidneys of male and female mice during NTN. To treat mice with estrogens, E2 pellets were implanted s.c. as described in 'Materials and Methods'. Kidneys were collected $30 \mathrm{~h}$ following NTS injection. Paraffin-embedded sections were stained for active caspase-3 as a measure of apoptosis and Poly (ADP-Ribose) Polymers (PARs) as a measure of PARP-1 activation and as a mediator of necrosis. Figure 5 a shows that NTS induced apoptosis only in males treated with E2, and therefore supports our in vitro observations. Figure $5 \mathrm{a}$ also shows that E2 inhibits caspase- 3 activation in females, which is in agreement with our in vitro results showing that E2 inhibits both apoptosis and necrosis in female cells (Figures $4 a-f)$. In line with the results in Figures $4 g$ and $h$, PARP-1 activation in males is inhibited by E2, whereas E2 had no effect on PARP-1 activation in females (Figure 5b). However, real-time PCR data showed that treatment of male mice with E2 significantly increased $E R \alpha$ expression (Figure 5c), whereas $E R \alpha$ expression in female mice was unaffected by additional E2 treatment (Figure $5 \mathrm{~d}$ ). The apparent differences in the ability of E2 to increase ER $\alpha$ between our in vitro and in vivo data could be due to a prolonged E2 treatment in vivo compared to a short-term treatment of cells in vitro.

\section{Discussion}

Our data suggest that cell death is influenced by the biological sex. One of the mechanisms is through the activation of PARP-1. Necrosis in males is PARP-1-dependent, with absence or inhibition of PARP-1 switching the inflammatory a
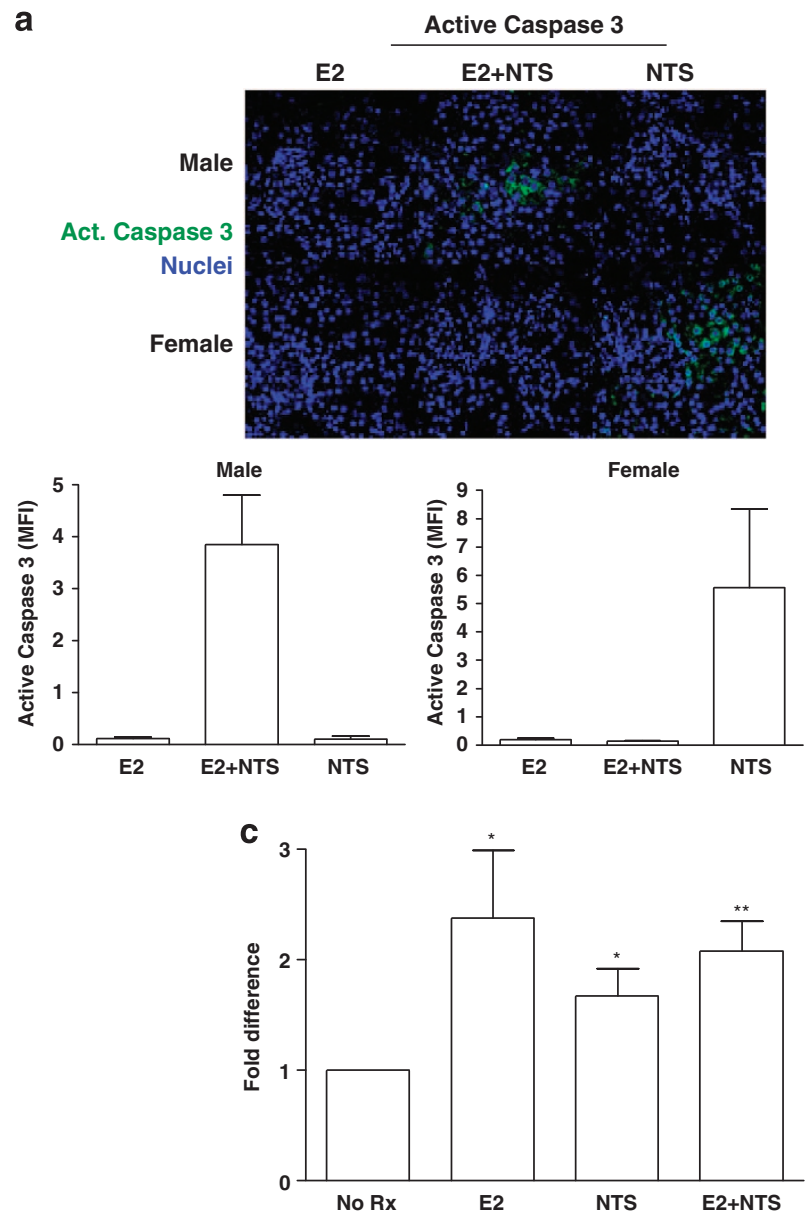

b
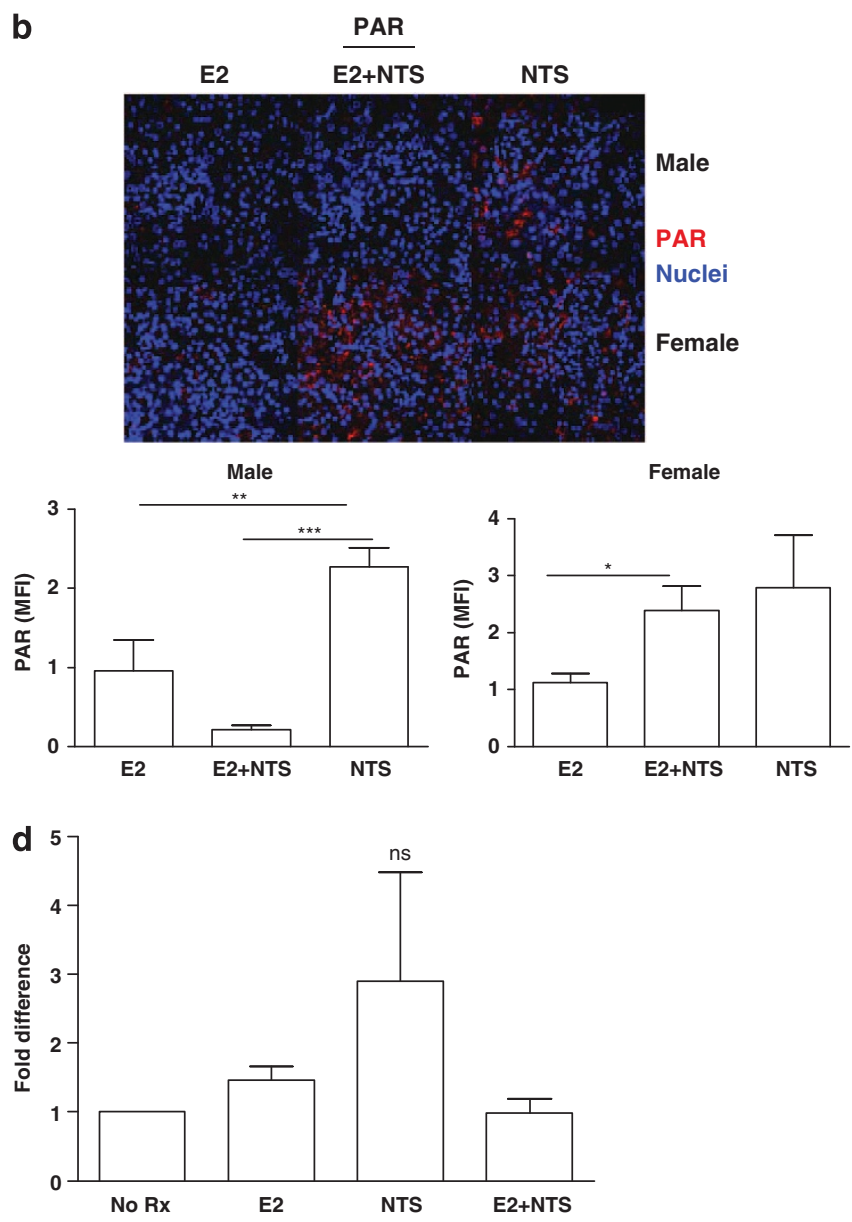

Figure 5 E2 treatment induces caspase-3 activation following NTN in male but not in female mice. To determine the role of E2 in the induction of cells death, we treated male or female mice with estrogens by implanting E2 pellets s.c. NTS $(6 \mathrm{ml} / \mathrm{kg})$ was injected 5 days following pellet implantation, and the kidneys were collected after $30 \mathrm{~h}$. Paraffin-embedded kidney sections were stained for active caspase-3 as a measure of apoptosis (a) or PAR as a measure of PARP-1 activation (b). Fluorescence intensities of red channel were measured using ImageJ software (lower panels). Fluorescence intensities of the secondary controls were subtracted from the stained sections. An average of 7-10 fields was calculated for each mouse. Data are mean \pm S.E.M., representative of six mice. The data show that E2 treatment of male mice results in increased apoptotic cell death and inhibition of necrosis following induction of nephritis. ER $\alpha \mathrm{mRNA}$ expression in the renal tissue from male (c) or female (d) mice was determined by quantitative PCR and normalized to mRNA levels in an untreated mouse of same sex. The results show that E2 treatment of males resulted in the upregulation of ER $\alpha$ in the kidney. The data are representative of six mice. ${ }^{*} P<0.05,{ }^{* \star} P<0.005,{ }^{* * *} P<0.0005$ 
cell death program to an anti-inflammatory apoptotic death program. In females, PARP-1 activation is independent of necrosis induction; however, estrogens have a survival role in these cells. The ability of estrogen to rescue female cells from death is independent of estrogen receptor expression, as ER $\alpha$ expression in male and female cells was similar. We propose that male cells are programmed to undergo necrosis, whereas female cells preferentially undergo apoptosis and that estrogens may have an important role in this sex-biased cell death.

The switch between necrosis and apoptosis upon PARP-1 inhibition was first reported in peroxinitrite-induced cell death in thymocytes. ${ }^{36} \mathrm{~A}$ similar switch has been reported in alkylating DNA damage-induced death in human tumor cell lines. ${ }^{37,38}$ Liu et al. ${ }^{37}$ suggested that ATP levels were a determining factor for the fate of the cell. Our data are consistent with the literature; however, in the present study we report a sex bias in the induction of necrosis by PARP-1. Specifically, we show that absence of PARP-1 induced apoptosis only in male cells.

Males and females respond differently to stress. Our data suggest that proclivity of male and female cells to different forms of cell death may contribute to the different responses of males and females to stress. We suggest PARP-1 as one of the mediators. PARP-1 is activated in both males and females (Figures 1 and 5b); however, the inhibition of PARP-1 in an inflammatory setting is protective only in males. ${ }^{22}$ Whether PARP-1 activation in females is a consequence of massive inflammatory stimulus and whether females have evolutionarily developed pathways of inducing necrosis independently of PARP-1 remains to be determined. In this study we show that E2 inhibits PARP-1 activation stimulated by $\mathrm{H}_{2} \mathrm{O}_{2}$ only in male cells. We hypothesize that due to higher levels of E2, females may have a different pathway for inducing necrosis independent of PARP-1 activation; however, the necrotic cell death pathway utilized by female cells is still unclear. PARP-1 activity has been shown to regulate basal and ligand-induced androgen receptor (AR) functions. ${ }^{39}$ Although signaling through AR has been shown to induce apoptosis, ${ }^{40-42}$ the exact mechanism of how AR regulates death is not clear. Moreover, the relevance of PARP-1 interaction with AR to regulate cell death is not yet known. Therefore, we cannot rule out a role for AR signaling in the sex bias we observe with PARP-1-induced cell death.

Extensive research has been focused on elucidating the role of sex hormones in sexual dimorphism observed in several diseases. The protection of females from inflammatory responses has been shown in several disease models. Exercise-induced muscle inflammatory response is less severe in women than in men, ${ }^{43}$ female rodents have improved hepatocellular function following trauma, and also improved survival in sepsis. However, chromosomal differences between males and females may also contribute to differences in their responses to stress. Male and female kidneys are known to be developmentally different. Females have more glomeruli per gram of kidney weight and lower renal plasma flow. ${ }^{44}$ Furthermore, a role for sex-chromosome component has been established in autoimmune diseases such as experimental autoimmune encephalomyelitis and lupus. ${ }^{29}$ The mosaic expression pattern of $\mathrm{X}$ chromosome genes in female is proposed to be protective. ${ }^{45}$ Females have two copies of $X$ chromosome, and therefore, two alleles for genes on $\mathrm{X}$ chromosome. The $\mathrm{X}$-chromosome inactivation is in a mosaic pattern, resulting in different cells having different alleles actively transcribed. A mutation in one allele produces less-prominent phenotypic changes due to expression of the normal allele in another cell. However, in males the same mutation is not compensated for by the second allele, which may be the reason for increased severity of diseases caused by a mutation in single $X$-linked gene, such as Fragile $X$ Syndrome and hemophilia.

Several cell death pathways such as the pathways regulated by mitogen-activated protein kinases (JNK, ERK and p38) are activated by ischemia/reperfusion and trauma hemorrhage, and are believed to be regulated by sex hormones. ${ }^{46-48}$ Estrogens are also protective in cardiomyocyte injury. Patten et al. ${ }^{49}$ showed that estrogen-mediated activation of the phospho-inositide-3 kinase (PI3K)-Akt pathway reduces cardiomyocyte apoptosis in vivo and in vitro. In this study we propose that the differences in the responses of males and females to stress may be due to inherent differences in susceptibility to undergo inflammatory necrosis. We further show that the responses of males and females to estrogen are different. It is likely that females have evolutionarily developed reduced sensitivity to estrogens and have developed alternative pathways to undergo necrotic cell death. The understanding of these pathways in females requires further studies. In line with this hypothesis, it would be interesting to determine the susceptibility to necrosis of cells from men with Klinefelter's syndrome (KS). KS (47, XXY) is the most common sex-chromosome disorder in man. ${ }^{50}$ Analysis of responses of cells from KS men to various death stimuli may explain whether a gene-dose effect is responsible for different susceptibilities of male and females to necrosis. Interestingly and pertinent to our observation, KS men with SLE have a milder disease and especially a milder form of nephritis, possibly due to a lesser activation of the necrotic pathway. ${ }^{51}$

Owing to differences in the responses to stimuli and mortality rates between males and females, there is a need for novel biomarkers and gender-based therapeutic approaches. Necrotic cell death is recognized as programmed and therefore potentially controllable. Our data suggest that males and females are prone to different types of cell death and this may explain how sex contributes to the frequency and severity of diseases such as lupus glomerulonephritis. Understanding the molecular pathways regulating tissue damage in males versus females will lead to tailored treatments for each gender.

\section{Materials and Methods}

Mice. 129sv, 129 PARP-1 - I - and C57/BL6 (B6) mice were obtained from Jackson Laboratories (Bar Harbor, ME, USA). B6. RIP-3 - / - mice were a kind gift from Dr. Dixit (Genentech) and were obtained from Fox Chase Cancer Center. Breeding colonies were maintained at Temple University in accordance with the guidelines of the University Laboratory Animal Resource Office of Temple University. All experimental procedures were conducted according to the guidelines of the Institutional Animal Care and Use Committee. NTN was induced, as described previously, by using a single injection of NTS. ${ }^{22}$ For estrogen treatment, $17 \beta$-estradiol (E2) pellets (Innovative Research of America, Sarasota, FL, USA) were implanted s.c. NTS $\left(6 \mathrm{mg} / \mathrm{ml}^{22}\right)$ was injected 5 days later 
and kidneys were harvested $30 \mathrm{~h}$ following NTS injection. Paraffin-embedded kidney sections from lupus-prone (NZWXBXSB)F1 mice were generously provided by Dr. A Davidson (Feinstein Institute, New York, NY, USA). In this model male mice develop a more severe renal disease than females. ${ }^{25}$ To accelerate lupus disease in females and obtain a comparable renal disease, (NZWXBXSB)F1 female mice were injected with an adenovirus expressing Interferon Alpha (AdvIFN $\alpha$ ) at 8 weeks of age. Kidneys were harvested at 22 weeks of age, as previously published. ${ }^{25}$ The (NZWXBXSB)F1 males were 18 weeks old. All mice had proteinuria at the time of tissue harvest.

BMDM cultures. BMDM were generated as described previously ${ }^{52}$ with the following modifications. Briefly, bone marrow cells were cultured in phenol red-free RPMI 1640 supplemented with 5\% FBS, recombinant mouse MCSF $(10 \mathrm{ng} / \mathrm{ml})$ and L929 conditioned medium (15\%). Non-adherent cells were collected after $24 \mathrm{~h}$ and cultured in Corning Ultra Low six-well plates (Corning Inc., Tewksbury, MA, USA) for 7-9 days. The medium was supplemented once on day 4 and every day after day 7. To deplete estrogens in the cultures, charcoal-dextran-treated fetal bovine serum was used for culturing BMDM and L929 cells.

Ex vivo cell death. Paraffin-embedded kidney sections were dewaxed with xylenes and rehydrated with decreasing concentrations of alcohol. Active caspase-3 was stained using a rabbit polyclonal antibody (BD Biosciences, San Jose, CA, USA) following heat-induced antigen retrieval. Poly (ADP-Ribose) polymers were stained, as described previously, ${ }^{22}$ using polyclonal anti-PAR antibody (Enzo Life Sciences Inc., Farmingdale, NY, USA). Images were acquired on Zeiss Meta laser scanning confocal microscope (Carl Zeiss Microscopy, LLC, Tornwood, NY, USA) equipped with Zen software. The data were analyzed by ImageJ software (http://rsbweb.nih.gov/ij/). The channels were separated (blue and green or blue and red). The mean fluorescence intensity (MFI) of each field was calculated for either red or green channel. The MFI for the sections with secondary antibody only were used for background calculations and were subtracted from the MFI values obtained from the sections stained with primary antibody. A minimum of 5-7 fields for each section/mouse were analyzed.

In vitro cell death. Cell death was induced by stimulating cells with $\mathrm{H}_{2} \mathrm{O}_{2}$. Following a 2-h stimulation, cells were harvested, stained with FITC-conjugated Annexin $\mathrm{V}$ and propidium iodide or 7AAD, and acquired on BD FACS Canto immediately. Apoptotic cells were defined as Annexin V single-positive, necrotic as Propidium lodide/7AAD-positive and live cells as Annexin V-negative Propidium lodide/7AAD-negative. ${ }^{53,54}$ Cells were stained for intracellular caspase-3 using $\mathrm{PE}$-conjugated active caspase-3 antibody (BD Biosciences) according to the manufacturer's instructions. For E2 treatment, cells were incubated with different concentrations of $\mathrm{E} 2$ for $3 \mathrm{~h}$ prior to stimulation with $\mathrm{H}_{2} \mathrm{O}_{2}$. For caspase-3 inhibition cells were pre-treated with Z-DEVD-FMK $(50 \mu \mathrm{m})$ for $30 \mathrm{~min}$ followed by further 2-h stimulation with $\mathrm{H}_{2} \mathrm{O}_{2}$. For microscopy, cells were labeled with DNA dye Hoechst 33342 during the $2-h$ stimulation with $\mathrm{H}_{2} \mathrm{O}_{2}$. Following stimulation, cells were fixed with $4 \%$ paraformaldehyde, and visualized with Zeiss Meta laser scanning confocal microscope. Cells were counted from 8-10 fields per condition (minimum of 100 cells) and the percentage of apoptotic and necrotic cells was calculated.

Western blotting. BMDMs were stimulated with $\mathrm{H}_{2} \mathrm{O}_{2}$ for $2 \mathrm{~h}$. Protein concentration in whole-cell lysates was determined by bicinchoninic acid (BCA) kit (Pierce Thermo Scientific, Rockford, IL, USA). Equal proteins were separated on $12 \%$ sodium dodecyl sulfate-polyacrylamide gel (SDS-PAGE) and transferred to nitrocellulose membrane. Anti-active caspase-3 antibody (Cell Signaling Technology, Inc., Danvers, MA, USA) was used to detect active caspase-3, and blots were developed using an ECL system Pierce Thermo Scientific. As a loading control, blots were re-probed with anti- $\beta$-actin antibody (Sigma-Aldrich, St. Louis, MO, USA). Band intensities were calculated using gel analyzer (ImageJ). The supernatants were collected and spun at 1500 r.p.m. for 10 min to remove cell remnants. Equal volumes of supernatants were concentrated using Amicon ultra concentrators (EMD Millipore, Billerica, MA, USA), separated using SDS-PAGE and transferred to nitrocellulose membrane. Anti-active caspase-3 antibody (Cell Signaling Technology, Inc.) was used to detect active caspase-3, and blots were developed using an ECL system (Pierce Thermo Scientific). As a positive control we induced apoptosis in Jurkat cells by stimulating with $4 \mu \mathrm{m}$ camptothecin for $3 \mathrm{~h}$. Whole-cell lysates of treated and untreated Jurkat cells were used for western blots as positive and negative controls for active caspase-3.
In vitro PARP-1 activity. PARP-1 activity in BMDMs was determined using a colorimetric assay, as described previously. ${ }^{55}$ Briefly, cells were seeded in a 96-well plate and stimulated with $500 \mu \mathrm{M} \mathrm{H}_{2} \mathrm{O}_{2}$. Following stimulation, the medium was replaced with PARP-1 reaction buffer ( $56 \mathrm{~mm}$ HEPES, $28 \mathrm{~mm} \mathrm{KCl}, 28 \mathrm{~mm}$ $\mathrm{NaCl}, 2 \mathrm{~mm} \mathrm{MgCl} 2, \mathrm{pH} 8.0,0.01 \%$ digitonin and $10 \mu \mathrm{m}$ biotinylated $\left.\mathrm{NAD}^{+}\right)$. The cells were fixed with pre-chilled $95 \%$ ethanol $(10 \mathrm{~min}$ at $-20 \mathrm{C})$, and endogenous peroxidase was blocked by $0.5 \% \mathrm{H}_{2} \mathrm{O}_{2}$ in methanol for $15 \mathrm{~min}$. Cells were washed once with $1 \times \mathrm{PBS}$ and blocked with $1 \%$ BSA/PBS. After $30 \mathrm{~min}$, BSA solution was aspirated and streptavidin-peroxidase (Jackson Immunoresearch Laboratories, West Grove, PA, USA) was added. Color was developed with TMB substrate (R\&D Systems Inc., Minneapolis, MN, USA). The optical density was measured with a microplate spectrophotometer. For E2 treatments, cells were incubated with different concentration of E2 for $3 \mathrm{~h}$ prior to stimulation.

Real-time PCR. Total RNA was isolated using Qiagen RNeasy kit (Qiagen, Valencia, CA, USA). RNA $(1 \mu \mathrm{g})$ was reverse-transcribed using Applied Biosystems high-capacity reverse-transcription kit (Applied Biosystems, Foster City, CA, USA). Estrogen Receptor $\alpha$ (Esr1) and Estrogen Receptor $\beta$ (Esr2) gene expression, normalized to GAPDH, was detected by real-time PCR using QuantiTect primer assays (Qiagen) and Eppendorf Realplex thermal cycler. The mRNA transcripts were quantified by standard curve method. RNA isolated from the uterus of a female mouse was used to generate the standard curve.

Statistical analysis. ANOVA and t-tests were performed using GraphPad Prism 4.0c software for Mac (GraphPad Software, Inc., La Jolla, CA, USA). Differences among groups were determined using the Tukey-Kramer post hoc test. Statistical significance was defined as $P<0.05$.

\section{Conflict of Interest}

The authors declare no conflict of interest.

Acknowledgements. We thank Dr. Anne Davidson for providing NZW/BXSB F1 kidneys and Genentech (San Francisco, CA, USA) for RIP-3 - / - mice. The authors would also like to thank Dr. PL Cohen for critically reading the manuscript. This work was supported by the Lupus Research Institute and NIH (R01 AR06156901A1) grants to RC.

1. Fairweather D, Frisancho-Kiss $S$, Rose NR. Sex differences in autoimmune disease from a pathological perspective. Am J Pathol 2008; 173: 600-609.

2. Gleicher N, Barad DH. Gender as risk factor for autoimmune diseases. J Autoimmun 2007; 28: 1-6.

3. Klein SL. Hormonal and immunological mechanisms mediating sex differences in parasite infection. Parasite Immunol 2004; 26: 247-264.

4. Lorenzo ME, Hodgson A, Robinson DP, Kaplan JB, Pekosz A, Klein SL. Antibody responses and cross protection against lethal influenza $A$ viruses differ between the sexes in C57BL/6 mice. Vaccine 2011; 29: 9246-9255.

5. Cihakova D, Talor MV, Barin JG, Baldeviano GC, Fairweather D, Rose NR et al. Sex differences in a murine model of Sjogren's syndrome. Ann NY Acad Sci 2009; 1173 378-383.

6. Myasoedova E, Crowson CS, Kremers HM, Therneau TM, Gabriel SE. Is the incidence of rheumatoid arthritis rising?: results from Olmsted County, Minnesota, 1955-2007. Arthritis Rheum 2010; 62: 1576-1582.

7. Giron-Gonzalez JA, Moral FJ, Elvira J, Garcia-Gil D, Guerrero F, Gavilan I et al. Consistent production of a higher TH1:TH2 cytokine ratio by stimulated T cells in men compared with women. Eur J Endocrinol 2000; 143: 31-36.

8. Bindl L, Buderus S, Dahlem P, Demirakca S, Goldner M, Huth R et al. Gender-based differences in children with sepsis and ARDS: the ESPNIC ARDS Database Group. Intensive Care Med 2003; 29: 1770-1773.

9. Moss M, Mannino DM. Race and gender differences in acute respiratory distress syndrome deaths in the United States: an analysis of multiple-cause mortality data (1979-1996). Crit Care Med 2002; 30: 1679-1685.

10. Sperry JL, Minei JP. Gender dimorphism following injury: making the connection from bench to bedside. J Leukoc Biol 2008; 83: 499-506

11. Barratt J, Feehally J. IgA nephropathy. J Am Soc Nephrol 2005; 16: 2088-2097.

12. Ward MM, Studenski S. Systemic lupus erythematosus in men: a multivariate analysis of gender differences in clinical manifestations. J Rheumatol 1990; 17: 220-224.

13. Lu LJ, Wallace DJ, Ishimori ML, Scofield RH, Weisman MH. Review: male systemic lupus erythematosus: a review of sex disparities in this disease. Lupus 2009; 19: 119-129.

14. de Carvalho JF, do Nascimento AP, Testagrossa LA, Barros RT, Bonfa E. Male gender results in more severe lupus nephritis. Rheumatol Int 2010; 30: 1311-1315. 
15. Du L, Bayir H, Lai Y, Zhang X, Kochanek PM, Watkins SC et al. Innate gender-based proclivity in response to cytotoxicity and programmed cell death pathway. $\mathrm{J}$ Biol Chem 2004; 279: 38563-38570.

16. Li H, Pin S, Zeng Z, Wang MM, Andreasson KA, McCullough LD. Sex differences in cell death. Ann Neurol 2005; 58: 317-321.

17. Edinger AL, Thompson CB. Death by design: apoptosis, necrosis and autophagy. Cur Opin Cell Biol 2004; 16: 663-669.

18. Zong WX, Thompson CB. Necrotic death as a cell fate. Genes Dev 2006; 20: 1-15

19. Vandenabeele P, Galluzzi L, Vanden Berghe T, Kroemer G. Molecular mechanisms of necroptosis: an ordered cellular explosion. Nat Rev Mol Cell Biol 2010; 11: 700-714.

20. Schreiber V, Dantzer F, Ame JC, de Murcia G. Poly(ADP-ribose): novel functions for an old molecule. Nat Rev Mol Cell Biol 2006; 7: 517-528.

21. Kirkland JB. Poly ADP-ribose polymerase-1 and health. Exp Biol Med (Maywood) 2010 235: 561-568.

22. Jog NR, Dinnall JA, Gallucci S, Madaio MP, Caricchio R. Poly(ADP-ribose) polymerase-1 regulates the progression of autoimmune nephritis in males by inducing necrotic cell death and modulating inflammation. J Immunol 2009; 182: 7297-7306.

23. Baba T, Shimizu T, Suzuki Y, Ogawara M, Isono K, Koseki H et al. Estrogen, insulin, and dietary signals cooperatively regulate longevity signals to enhance resistance to oxidative stress in mice. J Biol Chem 2005; 280: 16417-16426.

24. Mabley JG, Horvath EM, Murthy KG, Zsengeller Z, Vaslin A, Benko R et al. Gender differences in the endotoxin-induced inflammatory and vascular responses: potential role of poly(ADP-ribose) polymerase activation. J Pharmacol Exp Ther 2005; 315: 812-820.

25. Ramanujam M, Kahn P, Huang W, Tao H, Madaio MP, Factor SM et al. Interferon-alpha treatment of female (NZW x BXSB)F(1) mice mimics some but not all features associated with the Yaa mutation. Arthritis Rheum 2009; 60: 1096-1101.

26. Fu Y, Du Y, Mohan C. Experimental anti-GBM disease as a tool for studying spontaneous lupus nephritis. Clin Immunol 2007; 124: 109-118.

27. Du Y, Fu Y, Mohan C. Experimental anti-GBM nephritis as an analytical tool for studying spontaneous lupus nephritis. Arch Immunol Ther Exp (Warsz) 2008; 56: 31-40.

28. Lockshin MD. Nonhormonal explanations for sex discrepancy in human illness. Ann NY Acad Sci 2010; 1193: 22-24.

29. Smith-Bouvier DL, Divekar AA, Sasidhar M, Du S, Tiwari-Woodruff SK, King JK et al. A role for sex chromosome complement in the female bias in autoimmune disease. $J$ Exp Med 2008; 205: 1099-1108.

30. Hirt UA, Leist M. Rapid, noninflammatory and PS-dependent phagocytic clearance of necrotic cells. Cell Death Differ 2003; 10: 1156-1164.

31. Appelt U, Sheriff A, Gaipl US, Kalden JR, Voll RE, Herrmann M. Viable, apoptotic and necrotic monocytes expose phosphatidylserine: cooperative binding of the ligand Annexin $\mathrm{V}$ to dying but not viable cells and implications for PS-dependent clearance. Cell Death Differ 2005; 12: 194-196.

32. Degterev A, Hitomi J, Germscheid M, Ch'en IL, Korkina O, Teng X et al. Identification of RIP1 kinase as a specific cellular target of necrostatins. Nat Chem Biol 2008; 4: 313-321.

33. Vanden Berghe T, Vanlangenakker N, Parthoens E, Deckers W, Devos M, Festiens N et al. Necroptosis, necrosis and secondary necrosis converge on similar cellular disintegration features. Cell Death Differ 2010; 17: 922-930.

34. Straub RH. The complex role of estrogens in inflammation. Endocr Rev2007; 28: 521-574.

35. Hall JM, McDonnell DP. The estrogen receptor beta-isoform (ERbeta) of the human estrogen receptor modulates ERalpha transcriptional activity and is a key regulator of the cellular response to estrogens and antiestrogens. Endocrinology 1999; 140 5566-5578.

36. Virag L, Scott GS, Cuzzocrea S, Marmer D, Salzman AL, Szabo C. Peroxynitrite-induced thymocyte apoptosis: the role of caspases and poly (ADP-ribose) synthetase (PARS) activation. Immunology 1998; 94: 345-355.

37. Liu X, Luo X, Shi Y, Zhu GD, Penning T, Giranda VL et al. Poly (ADP-ribose) polymerase activity regulates apoptosis in HeLa cells after alkylating DNA damage. Cancer Biol The 2008; 7: 934-941
38. Tentori L, Balduzzi A, Portarena I, Levati L, Vernole P, Gold B et al. Poly (ADP-ribose) polymerase inhibitor increases apoptosis and reduces necrosis induced by a DNA minor groove binding methyl sulfonate ester. Cell Death Differ 2001; 8: 817-828.

39. Schiewer MJ, Goodwin JF, Han S, Brenner JC, Augello MA, Dean JL et al. Dual roles of PARP-1 promote cancer growth and progression. Cancer Discov 2012; 2 1134-1149.

40. Heisler LE, Evangelou A, Lew AM, Trachtenberg J, Elsholtz HP, Brown TJ. Androgen-dependent cell cycle arrest and apoptotic death in PC-3 prostatic cell cultures expressing a full-length human androgen receptor. Mol Cell Endocrinol 1997; 126: 59-73.

41. Olsen NJ, Viselli SM, Fan J, Kovacs WJ. Androgens accelerate thymocyte apoptosis. Endocrinology 1998; 139: 748-752.

42. Frezza M, Yang H, Dou QP. Modulation of the tumor cell death pathway by androgen receptor in response to cytotoxic stimuli. J Cell Physiol 2012; 226: 2731-2739.

43. Timmons BW. Exercise and the cytokine balance: a paediatric perspective. J Sports Sci 2006; 24: 1-2

44. Munger K, Baylis C. Sex differences in renal hemodynamics in rats. Am J Physiol 1988; 254(2 Pt 2): F223-F231.

45. Migeon BR. The role of $X$ inactivation and cellular mosaicism in women's health and sex-specific diseases. JAMA 2006; 295: 1428-1433.

46. Jover-Mengual T, Zukin RS, Etgen AM. MAPK signaling is critical to estradiol protection of CA1 neurons in global ischemia. Endocrinology 2007; 148: 1131-1143.

47. Vilatoba M, Eckstein C, Bilbao G, Frennete L, Eckhoff DE, Contreras JL. 17beta-estradiol differentially activates mitogen-activated protein-kinases and improves survival following reperfusion injury of reduced-size liver in mice. Transplant Proc 2005; 37 399-403.

48. Stice JP, Mbai FN, Chen L, Knowlton AA. Rapid activation of nuclear factor kappab by 17beta-estradiol and selective estrogen receptor modulators: pathways mediating cellular protection. Shock 2012; 38: 128-136.

49. Patten RD, Pourati I, Aronovitz MJ, Baur J, Celestin F, Chen X et al. 17beta-estradiol reduces cardiomyocyte apoptosis in vivo and in vitro via activation of phospho-inositide-3 kinase/Akt signaling. Circ Res 2004; 95: 692-699.

50. Lanfranco F, Kamischke A, Zitzmann M, Nieschlag E. Klinefelter's syndrome. Lancet 2004; 364: 273-283.

51. Dillon S, Aggarwal R, Harding JW, Li LJ, Weissman MH, Li S et al. Klinefelter's syndrome (47, XXY) among men with systemic lupus erythematosus. Acta Paediatr 2011; 100: $819-823$

52. Stout RD, Jiang C, Matta B, Tietzel I, Watkins SK, Suttles J. Macrophages sequentially change their functional phenotype in response to changes in microenvironmental influences. J Immunol 2005; 175: 342-349.

53. Caricchio R, McPhie L, Cohen PL. Ultraviolet B radiation-induced cell death: critical role of ultraviolet dose in inflammation and lupus autoantigen redistribution. J Immunol 2003; 171 5778-5786.

54. Fiorillo C, Ponziani V, Giannini L, Cecchi C, Celli A, Nassi N et al. Protective effects of the PARP-1 inhibitor PJ34 in hypoxic-reoxygenated cardiomyoblasts. Cell Mol Life Sci 2006; 63: 3061-3071.

55. Bakondi E, Bai P, Szabo EE, Hunyadi J, Gergely P, Szabo C et al. Detection of poly(ADPribose) polymerase activation in oxidatively stressed cells and tissues using biotinylated NAD substrate. J Histochem Cytochem 2002; 50: 91-98.

(c) (3) (2) Cell Death and Disease is an open-access journal published by Nature Publishing Group. This work is licensed under a Creative Commons Attribution-NonCommercialShareAlike 3.0 Unported License. To view a copy of this license, visit http://creativecommons.org/licenses/by-nc-sa/3.0/ 